\title{
Enumeration And Characterization Of Circulating Tumor Cells And Its Application In Advanced Gastric Cancer
}

\section{Boran Cheng ${ }^{l, *}$ Gangling Tong ${ }^{1, *}$ \\ Xuan Wu (iD ${ }^{1, *}$ \\ Wenwu Cai ${ }^{2, *}$ \\ Zhu $\mathrm{Li}^{1}{ }^{1} *$ \\ Zhongyi Tong ${ }^{2}$ \\ Lirui $\mathrm{He}^{\prime}$ \\ Shaokang Yu' \\ Shubin Wang'}

'Department of Oncology, Peking University Shenzhen Hospital, Shenzhen, Guangdong Province 5I8036, People's Republic of China; ${ }^{2}$ Department of General Surgery, The Second Xiangya Hospital of Central South University, Changsha, Hunan Province 4I00II,

People's Republic of China

*These authors contributed equally to this work
This article was published in the following Dove Press journal: OncoTargets and Therapy

Background: Advanced gastric cancer (aGC) has a high global incidence and a high mortality rate and because of its high malignancy and heterogeneity, the existing methods for prognosis are limited, and a new treatment model is necessary. Circulating tumor cells (CTCs) could be considered as a "liquid biopsy" for tumor diagnosis and for monitoring treatment responses and predicting clinical outcome. Clinical studies support the efficacy of programmed cell death 1 (PD-1) immunotherapy in a subset of aGC.

Methods: Cell capture efficiency, as described by the CanPatrol CTC enrichment technique, was validated using artificial blood samples as well as blood samples from 32 aGC patients. Clinicopathologic data of patients were collected from the hospital information system. We used CanPatrol for CTC isolation, classification, and clinical analysis.

Results: A cell capture efficiency of $>80 \%$ was achieved. Significant correlation was observed between CTC enumeration and clinicopathology parameters, including the Lauren classification $(\mathrm{r}=0.470, \mathrm{P}=0.008)$, perineural invasion $(\mathrm{r}=0.393, \mathrm{P}=0.029)$, TNM stage $(\mathrm{r}=0.740, \mathrm{P}<0.001)$, and $\mathrm{Ki}-67$ level $(\mathrm{r}=0.510, \mathrm{P}=0.005)$. When compared to the traditional methods, monitoring CTC subtypes exhibits higher sensitivity of evaluating the disease status. Enumeration of epithelial CTC subset and its relative abundance in the total CTC pool are highly correlated with clinical efficacy. CTC programmed cell death ligand-1 (PD-L1) could be successfully detected for immunotherapy in addition to PD-L1 immunohistochemistry and microsatellite instability.

Conclusion: We provide a new method that allows for the simple and effective detection of CTCs in aGC. It has potential clinical applications in monitoring prognosis and guiding future individualized immunotherapy.

Keywords: CTC enumeration, clinical response prediction, subgroup analysis, immune checkpoint therapy

\section{Introduction}

Gastric cancer (GC) is one of the most common malignancies and the second leading cause of cancer-related deaths worldwide. ${ }^{1-4}$ The early clinical symptoms of GC are insidious and atypical. Hence, at the time of consultation, more than $60 \%$ of the patients are found to have locally advanced or advanced cancer. The 5-year survival rate is only $2-15 \%{ }^{5}$ Patients with unresectable advanced and recurrent gastric cancer have poor prognosis, in spite of recent advances in chemotherapy, including the use of molecular targeted drugs such as trastuzumab and ramucirumab. ${ }^{6}$ Therefore, new therapeutic strategies based on new antineoplastic drugs are important for the treatment of advanced gastric cancer (aGC).
Wang

Department of Oncology, Peking

University Shenzhen Hospital, No. I 120,

Lianhua Road, Futian District, Shenzhen,

Guangdong Province 518036, People's

Republic of China

Tel +86 755 । $35-3093-5356$

Fax +86 755-8392-3333

Email BRcheng@whu.edu.cn ;

Shubinwang@aliyun.com 
Immune checkpoint blockade, as a new molecular targeted therapy, has attracted attention in patients with melanoma, ${ }^{7,8}$ lung cancer, ${ }^{9,10}$ gastric cancer, ${ }^{11,12}$, and other malignant tumors. ${ }^{13,14}$ However, clinical trials with PD-1 monoclonal antibodies such as pembrolizumab or nivolumab have shown a fairly wide response rate (10-26\%) in GC patients without selective biomarkers. ${ }^{15,16}$ A phase III study in the Asian population ATTRACTION-02 (NCT02267343) showed that the median survival time of patients treated with nivolumab was only extended from 4.1 months to 5.3 months. ${ }^{16,17}$ In another study, KEYNOTE-059, the effective rate of pembrolizumab monotherapy was found to be only $11.5 \% .{ }^{15} \mathrm{Hence}$, in order to enhance the efficacy of anti-PD-1 therapy in $\mathrm{GC}$, identification of predictive biomarkers and screening of the target population is urgently needed.

Circulating tumor cells (CTCs), regarded as "liquid biopsy", ${ }^{18}$ provide alternative access to solid tumors. This enables serial sampling of the tumor and thereby allows us to study the tumor variations throughout the treatment regime, which in turn is crucial for the discovery of new drugs and personalized therapy. ${ }^{19}$ Noninvasive liquid biopsy using blood specimens is clinically attractive because it is a simple sampling tool offering reproducible sampling and is more representative of the entire body state, thus overcoming the challenge of accurately representing the heterogeneity of tumors. ${ }^{20,21}$ However, detection and characterization of CTCs are still technically challenging because these cells are extremely rare and mixed with a large number of hematologic cells. ${ }^{19,22}$ We have developed a series of CTC capture platforms and successfully used them in clinical settings. ${ }^{23-27}$ But some limitations of the above methods (i.e., complex procedures, loss of CTC subpopulations) have restricted its further clinical use. In this study, we use $\mathrm{CanPatrol}^{\circledR}$, a CONFORMITE EUROPEENNE (CE)-certified CTC enrichment technique, ${ }^{28}$ for rare cancer cell isolation and their analysis in a clinical setting. Results showed that the capture efficiency of $>80 \%$ can be achieved through the use of CanPatrol. The five-color immunocytochemistry method was used to identify and enumerate the different CTC subsets, including fluorescently labeled EpCAM/cytokeratin, antiCD45, PD-L1, vimentin, and DAPI-stained nuclei. Given the high sensitivity and subgroup analysis capability of this method, we validated the ability of isolation and enumeration of CTCs in 32 aGC patients and tested its potential utility in monitoring a clinical response to treatment.

In this study, we aimed to evaluate the prognostic value of cancer cell counting during anticancer treatment in patients with GC. In addition, in order to better select GC patients who might benefit most from immune checkpoint therapy, we investigated the relationship between specific CTC subtypes and the efficacy of immunotherapy, as well as the change of CTC phenotype during chemotherapy.

\section{Materials And Methods Cell Lines And Patient Samples}

The gastric carcinoma cell line (MGC803), hepatocellular cancer cell line (HepG2) were harvested from Hubei Key Laboratory of Tumor Biological Behaviors (Hubei, China) under Institutional Review Board (IRB) protocol of Peking University Shenzhen Hospital. Cells were cultured in DMEM (BD Biosciences, San Jose, CA, USA) and supplemented with $10 \%$ FBS (Sigma-Aldrich Co. St Louis, MO, USA) and $1 \%$ penicillin/streptomycin solution at $37^{\circ}$ $\mathrm{C}$ under a humidified $5 \% \mathrm{CO}_{2}$ atmosphere.

With Eastern Cooperative Oncology Group Performance Status (ECOG-PS) $\leq 1$, patients of any age with unresectable advanced or metastatic gastric cancer were eligible for the study. Cancer patients' blood samples were obtained from the Department of Oncology, Peking University Shenzhen Hospital according to IRB protocol. Tissue specimens and blood samples were obtained from the Department of General Surgery, The Second Xiangya Hospital of Central South University under a separate IRB-approved protocol. All patients gave their written informed consent to participate in this study, and the research was approved by the Ethics Committee. All blood and tissue specimens were collected into anticoagulant tubes and sterile tubes (Wuxi Naisi Medical Technology Co, Ltd, Wuxi, China), respectively.

\section{Cell Classification And Enumeration}

First, according to the previously established method, ${ }^{28}$ CTCs were captured by the CanPatrol CTC enrichment technique. Then, a multiplex RNA in situ hybridization (RNA-ISH) assay based on branched DNA (bDNA) signal amplification was applied to classify and enumerate CTCs. PD-L1 (Abcam, Cambridge, MA, USA), leukocyte biomarker (CD45, BD Biosciences), epithelial biomarkers (EpCAM and CK8/18/ 19, R\&B systems, MN), and mesenchymal biomarkers (Vimentin and Twist, R\&B Systems, MN, USA) were used to identify and characterize CTC subsets. Five-color fluorescently labeled probes were added and incubated with cells. The sequences of the capture probes and bDNA signal amplification probes had been reported earlier and were synthesized by Invitrogen (Shanghai, China). ${ }^{28}$ The cell nuclei were stained with DAPI (Sigma), and the cells were analyzed with 
an automatic Axio Imager Z2 fluorescence microscope (Zeiss, Carl Zeiss Meditec AG, Jena, Germany). Finally, epithelial and mesenchymal biomarkers present red and green fluorescence, respectively. PD-L1 and CD45 present violet and bright blue, respectively.

\section{PD-LI IHC, CTC-PDLI, And MSI Status Determination}

Tissue microarray (TAM) was prepared and detected for the expression of PD-L1, MLH1 by immunohistochemical method in Department of Pathology, The Second Xiangya Hospital of Central South University. Anti-human PD-L1 $\mathrm{mAb}$ and anti-MLH1 mAb were purchased from Abcam. DAB kit was supplied by Beijing Suolaibao Technology Co., Ltd $\square$ Beijng, China. Tissue microarray was put into oven and waxed at $60^{\circ} \mathrm{C}$ for $1 \mathrm{hr}$. After dewaxing, EDTA solution was used to repair the antigen, and then the chip was put into endogenous peroxidase blocker to block for 15 mins. The antibody was incubated at room temperature according to the recommended concentration of the antibody instructions. Finally, the DAB kit was used to color and hematoxylin was used to re-stain. Results were statistically analyzed with clinic response.

\section{PD-LI IHC}

Five high-power visual fields $(\times 200$ times $)$ were randomly selected in tumor cells and tumor stroma with yellow to brown granules in the cytoplasm or cell membrane, respectively. The scoring criteria of staining intensity of positive cells were as follows: no staining was 0 points. The scores of light yellow, light brown, and brown were 1, 2, and 3, respectively, and the scores of positive cell density were as follows: the number of positive cells $\leq 70 \%$ was 1 point, $71 \%$ $\sim 80 \%$ was 2 points, $81 \%-90 \%$ was 3 points, and $>90 \%$ was 4 points. Multiplication of cell staining intensity and density scores $\geq 6$ scores as positive expression.

\section{CTC-PD-LI}

The proportion of PD-L1-positive cells in total CTCs in peripheral blood of $5 \mathrm{~mL}$ gastric cancer patients was counted. Positive score criteria were 0 for nonpositive cells, 1 for positive cells $(<20 \%), 2$ for positive cells $(20 \% \sim 50 \%)$, and 3 for positive cells $(>50 \%)$. The final score was $(\geq 2)$ for CTC-PD-L1-positive expression.

\section{MSI}

Each slice was randomly selected from five high-power visual fields with high positive rate. 100 cells were counted in each visual field. Positive score criteria were
0 for nonpositive cells, 1 for positive cells ( $\leq 10 \%), 2$ for positive cells $(10 \sim 50 \%), 3$ for positive cells $(50 \sim 80 \%)$, and 4 for positive cells $(>80 \%)$. The grading standard of dyeing strength was 0 for colorless, 1 for light yellow, 2 for brown, and 3 for brown. The final score was multiplied by the two scores. The final score was 0 for protein deletion and $(\geq 2)$ for protein-positive expression.

\section{Data Collection}

Clinicopathologic data of patients were collected from hospital information system, including gender, age, Lauren classification, differentiation degree, lymphovascular invasion, perineural invasion, tumor depth, lymph node status, metastasis status, TNM stage and the level of $\mathrm{Ki}-$ 67, tumor markers, smoking, and alcohol history. The clinicopathological classifications of cancers were determined according to the 7 th edition of the American Joint Committee on Cancer/International Union Against Cancer TNM classification system. ${ }^{29}$

\section{Statistical Analyses}

Categorical data displayed in a contingency table were analyzed using Fisher's exact test. Continuous data were analyzed by using the Mann-Whitney test and Kruskal-Wallis test. Spearman rank correlation analysis was used for nonparametric correlation analysis. All statistical analyses were carried out with the IBM SPSS statistical software package (IBM Inc.). $\mathrm{P}<0.05$ was considered statistically significant.

\section{Results And Discussion}

In order to study the cell capture efficiency using the CanPatrol CTC enrichment technique, a series of artificial blood samples were prepared by spiking MGC803/HepG2 cells into blood concentrations of approximately 10, 50, 100 , and 200 cells $5 \mathrm{~mL}^{-1}$. The assays were repeated 6 times for each sample. The results indicate that the average recovery for each sample was at least $80 \%$ and ranged from $80 \%$ to $90 \%$ (Figure $1 \mathrm{~A}$ and $\mathrm{B}$ ). These results represent a vast improvement in capture efficiency and stability compared to our previously established methods. ${ }^{24-26}$

To validate the clinical utility of this technique, $5 \mathrm{~mL}$ of the peripheral blood sample from aGC patients were introduced into CanPatrol for isolating and identifying CTCs. Captured cells were stained with the following: Alexa Fluor 594-labeled EpCAM/cytokeratin (epithelial marker, red), Alexa Fluor 488-labeled Vimentin/Twist (mesenchymal marker, green), Alexa Fluor 647-labeled CD45 (leukocyte marker, bright blue), and DAPI. Based on the staining, 
a

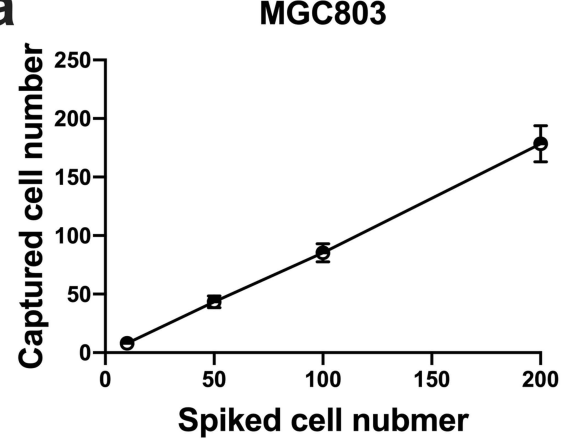

b

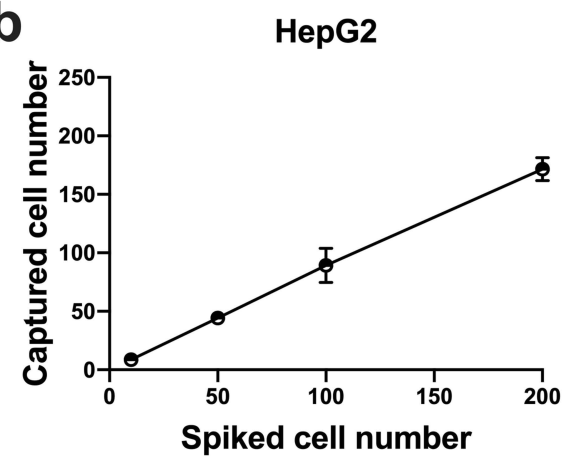

C

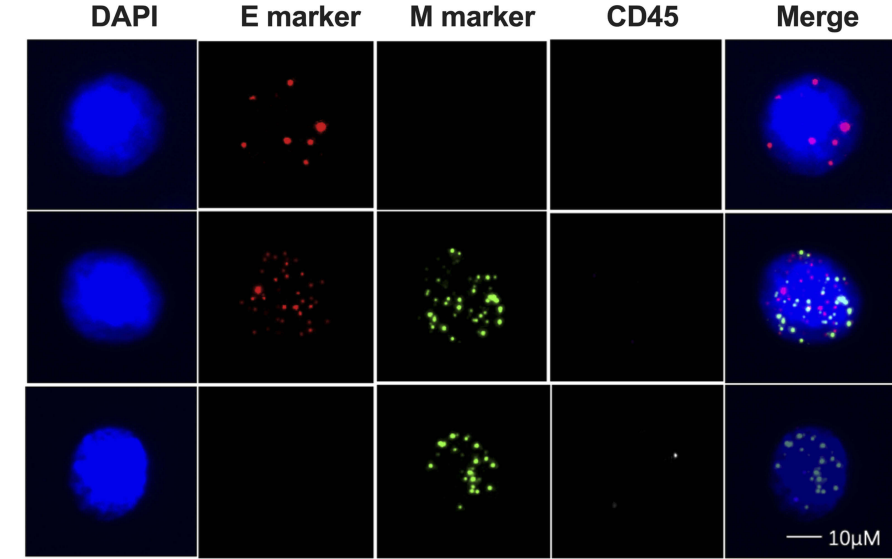

Epithelial CTCs

Hybrid CTCs

Mesenchymal CTCs

Figure I Capture efficiencies of two different cell lines ((A) MGC803 and (B) HepG2) were validated by CanPatrol ${ }^{\circledR}$. (C) Fluorescent micrographs of CTCs captured from whole blood samples collected from GC patients. The four-color immunocytochemistry method was used to identify and enumerate CTCs from nonspecifically captured WBCs, including Alexa Fluor 594 labeled EpCAM/Cytokeratin (epithelial markers, red), Alexa Fluor 488-labeled Vimentin/Twist (mesenchymal markers, green), Alexa Fluor 647-labeled CD45 (leukocyte markers, bright blue) and DAPI nuclear staining.

Abbreviations: CTCs, circulating tumor cells; GC, gastric cancer; WBC, white blood cell.

CTCs were divided into three types, namely epithelial CTCs (E+/M-/CD45-), hybrid epithelial/mesenchymal CTCs (E+/M $+/ \mathrm{CD} 45-)$, and mesenchymal CTCs (E-/M+/CD45-) (Figure 1C). Compared with the previous immunostaining method, ${ }^{27}$ this method offers higher sensitivity and lower background noise. Epithelial-mesenchymal transformation (EMT) plays an important role in cancer metastasis and drug resistance. Classification of CTC according to its EMT phenotype, which can be achieved by this method, is helpful for identifying the most aggressive CTC subsets and providing data for clinical application.

To study the clinical significance of CTCs in diagnosis, blood samples from $32 \mathrm{aGC}$ patients were detected by this technique. As shown in Figure 2A, captured CTCs were stained with EpCAM, vimentin, PD-L1, and DAPI. Fluorescence microscopy was used to distinguish specifically captured CTCs $(\mathrm{E} \pm / \mathrm{M} \mp / \mathrm{CD} 45-\mathrm{PD}-\mathrm{L} 1 \pm / \mathrm{DAPI}+)$ from nonspecifically immobilized WBCs (E-/M-/CD45+/DAPI + ) by comparing fluorescence and size (CTCs: $10-30 \mu \mathrm{m} ; 1$ WBCs: $<15 \mu \mathrm{m}$ ). The results of cell enumeration and clinical characteristics are shown in Figure 2B and Table 1. Cancer cells captured from the GC patients had total counts ranging from 0 to 37 per $5 \mathrm{~mL}$. In addition, the counts of epithelial CTCs ranged from 0 to 7, hybrid CTCs ranged from 0 to 34, and mesenchymal CTCs range from 0 to 9 , respectively. To further evaluate the correlation of CTCs with the clinical characteristics of GC patients, a statistical analysis was performed. The result indicated that both the CTC count and positive expression rate were significantly associated with Lauren classification (Figure 2C), perineural invasion (Figure 2D), M stage (Figure 2E), and Ki-67 level (Figure 2F). Spearman rank correlation analysis showed that CTC counts were highly correlated with $\mathrm{N}$ stage $(\mathrm{r}=0.379$, $\mathrm{P}=0.033)$ and TNM stage $(\mathrm{r}=0.740, \mathrm{P}<0.001)$ as shown in Table 2. Moreover, there was a significant difference in the level of carcinoembryonic antigen (CEA) and carbohydrate antigen-199 (CA199) between the positive and negative groups of CTCs (Table 3). Both positivity rate and CTC count were positively correlated with Lauren classification. This result may suggest that GC patients with diffuse type 

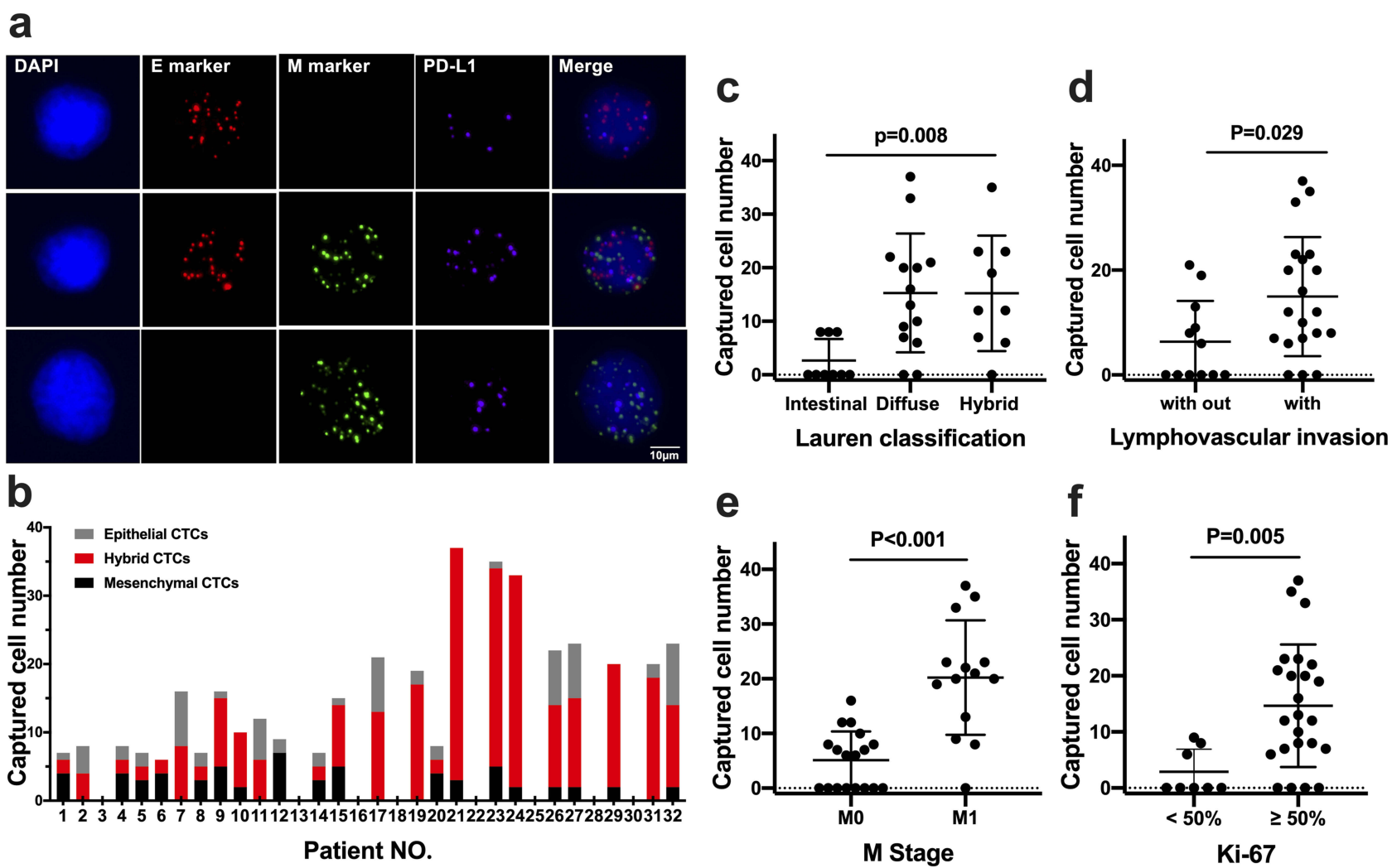

Figure 2 (A) CTCs identified from a GC patient. Immunocytochemistry method based on EpCAM/cytokeratin (E markers, red), Vimentin/Twist (M markers, green), PD-LI (violet) as well as DAPI nuclear staining. (B) CTC enumeration results obtained from 32 cancer patients. Scatter plot for CTC number of GC patients (C) with different Lauren classification, (D) with and without lymphovascular invasion, (E) different M stage, and (F) expression of Ki-67, each dot stands for one GC patient. The error bar represents $\mathrm{SE}$ of the mean.

Abbreviations: CTCs, circulating tumor cells; GC, gastric cancer; WBC, white blood cell; PD-LI, programmed cell death-ligand I.

had a worse prognosis than patients with intestinal type because there were more tumor cells in the peripheral blood, and they were more prone to metastasis. The correlation between vascular invasion and CTC also needs to be discussed. Vascular invasion has been confirmed to be an important prognostic factor for GC. As the primary tumors continue to increase in size and compress the surrounding blood vessels, some tumor cells enter the blood circulation through the fissures in the blood vessel wall to form CTCs, and some tumor cells passively migrate into the circulation through protein hydrolysis or other structural changes in the tumor microenvironment. Because CTCs shed from the primary tumor into the blood and maintain the specific phenotype of the tumor, it is feasible to enrich CTCs by detecting specific molecular markers. However, our result showed that both the positivity rate and CTC count in patients with vascular invasion were higher than those in patients without vascular invasion, which suggests that vascular invasion may also be important for tumor cells to enter the blood circulation. This could lead to distant metastasis. Hence, CTCs could be used in conjunction with TNM stage and Ki-67 to evaluate the degree of disease progression and malignancy, respectively.

To further evaluate the predictors of response with different CTC subtypes, some patients with mesenchymal CTCs (E-/M+/CD45-) were dynamically monitored during the therapeutic process. CTCs from these patients were synchronously compared with serum tumor marker levels and computed tomogram (CT) examination results according to Response Evaluation Criteria in Solid Tumors (RECIST). As shown in Figure 3A, a patient who received the SOX treatment regime (oxaliplatin plus S-1) showed a decline in $\mathrm{E}+\mathrm{CTC}$ numbers, and its relative abundance in the total CTC population paralleled effective therapeutic interventions. With clinical treatments, the E+CTC count of this patient declined along with shrinking of tumor burden (PR). After 3 months, during maintenance chemotherapy with SOX, the tumor progressed (PD) and the E + CTC count increased rapidly, while the total CTC and serum CEA concentrations were both unaltered. As shown in Figure 3B, another phenomenon was observed during 
Table I Gastric Cancer Patient Characteristics $(n=32)$

\begin{tabular}{|c|c|c|c|c|c|}
\hline Group & Patients & Percentage & Count Of CTCs+ Patients (n) & Count Of CTCs (mean \pm SD) & CTCs Positive Rate (\%) \\
\hline \multicolumn{6}{|l|}{ Sex } \\
\hline Male & 22 & 68.8 & 15 & $7.38 \pm 7.58$ & 68.2 \\
\hline Female & 10 & 31.2 & 8 & $7.38 \pm 7.58$ & 80.0 \\
\hline \multicolumn{6}{|l|}{ Age (years) } \\
\hline$\geq 60$ & 10 & 68.8 & 9 & $15.00 \pm 7.78$ & 90 \\
\hline$<60$ & 22 & 31.2 & 14 & $10.23 \pm 11.93$ & 63.6 \\
\hline \multicolumn{6}{|c|}{ Lauren classification } \\
\hline $\begin{array}{l}\text { Intestinal } \\
\text { type }\end{array}$ & 9 & 28.1 & 3 & $2.67 \pm 4.00$ & 33.3 \\
\hline Diffuse type & 14 & 43.8 & 12 & $15.29 \pm 11.10$ & 85.7 \\
\hline Hybrid type & 9 & 28.1 & 8 & $15.22 \pm 10.79$ & 88.9 \\
\hline \multicolumn{6}{|l|}{ Differentiation } \\
\hline Well & 3 & 9.4 & 2 & $13.00 \pm 11.27$ & 66.7 \\
\hline Moderate & 12 & 37.5 & 11 & $14.50 \pm 9.88$ & 91.7 \\
\hline Poor & 17 & 53.1 & 10 & $9.53 \pm 11.68$ & 58.8 \\
\hline \multicolumn{6}{|c|}{ Perineural invasion } \\
\hline No & 19 & 59.4 & 11 & $10.26 \pm 12.07$ & 57.9 \\
\hline Yes & 13 & 40.6 & 12 & $13.85 \pm 8.97$ & 92.3 \\
\hline \multicolumn{6}{|c|}{ Lymphovascular invasion } \\
\hline No & 11 & 34.4 & 5 & $6.33 \pm 7.81$ & 45.5 \\
\hline Yes & 21 & 65.6 & 18 & $14.95 \pm 11.38$ & 85.7 \\
\hline \multicolumn{6}{|l|}{ T stage } \\
\hline TI-2 & 6 & 18.8 & 4 & $7.83 \pm 8.16$ & 66.7 \\
\hline T3-4 & 26 & 81.2 & 19 & $12.62 \pm 11.39$ & 73.1 \\
\hline \multicolumn{6}{|l|}{$\mathrm{N}$ stage } \\
\hline No-I & 11 & 34.4 & 5 & $7.27 \pm 11.17$ & 45.5 \\
\hline N2-3 & 21 & 65.6 & 18 & $14.05 \pm 10.27$ & 85.7 \\
\hline \multicolumn{6}{|l|}{ M stage } \\
\hline Mo & 18 & 56.2 & 10 & $5.11 \pm 5.27$ & 55.6 \\
\hline MI & 14 & 43.8 & 13 & $20.21 \pm 10.45$ & 92.9 \\
\hline \multicolumn{6}{|l|}{ TNM stage } \\
\hline Stage II & 6 & 18.8 & 2 & $2.00 \pm 3.10$ & 33.3 \\
\hline Stage III & 12 & 37.5 & 8 & $6.67 \pm 5.53$ & 66.7 \\
\hline Stage IV & 14 & 43.8 & 13 & $20.21 \pm 10.45$ & 92.9 \\
\hline \multicolumn{6}{|l|}{ Ki-67 } \\
\hline$\geq 50 \%$ & 24 & 75 & 20 & $2.88 \pm 4.05$ & 83.3 \\
\hline$<50 \%$ & 8 & 25 & 3 & $14.67 \pm 10.91$ & 37.5 \\
\hline \multicolumn{6}{|l|}{ Smoking history } \\
\hline Yes & 13 & 40.6 & 8 & $10.38 \pm 11.13$ & 61.5 \\
\hline
\end{tabular}

(Continued) 
Table I (Continued).

\begin{tabular}{|c|c|c|c|c|c|}
\hline Group & Patients & Percentage & Count Of CTCs+ Patients (n) & Count Of CTCs (mean \pm SD) & CTCs Positive Rate (\%) \\
\hline No & 19 & 59.4 & 15 & $12.63 \pm 10.97$ & 78.9 \\
\hline \multicolumn{6}{|c|}{ Alcohol history } \\
\hline Yes & II & 34.4 & 10 & $12.45 \pm 9.84$ & 90.9 \\
\hline No & 21 & 65.6 & 13 & $11.33 \pm 11.64$ & 61.9 \\
\hline
\end{tabular}

Table 2 The Relationship Of CTCs And Clinicopathological Variables Of Gastric Cancer Patients

\begin{tabular}{|l|l|l|l|}
\hline \multirow{2}{*}{ Variable } & \multirow{2}{*}{$\begin{array}{l}\text { CTCs Positive } \\
\text { Rate } \\
\end{array}$} & $\mathbf{P}^{\mathbf{a}}$ & \multicolumn{2}{l|}{ Count Of CTCs } \\
\cline { 3 - 4 } & & $\mathbf{P}^{\mathbf{b}}$ & $\mathbf{P}^{\mathbf{c}}$ \\
\hline Sex & 0.155 & 0.696 & $0.703, r=0.070$ \\
Age & 1.000 & 0.064 & $0.063, r=0.333$ \\
Lauren & 0.010 & 0.008 & $0.007, r=0.470$ \\
classification & & & \\
Differentiation & 0.150 & 0.266 & $0.152, r=0.259$ \\
Perineural & 0.050 & 0.180 & $0.185, r=0.241$ \\
invasion & & & \\
Lymphovascular & 0.035 & 0.029 & $0.026, r=0.393$ \\
invasion & & & \\
T stage & 1.000 & 0.420 & $0.429, r=0.145$ \\
N stage & 0.035 & 0.035 & $0.033, r=0.379$ \\
M stage & 0.044 & $<0.001$ & $<0.001, r=0.704$ \\
TNM stage & 0.022 & $<0.001$ & $<0.001, r=0.740$ \\
Ki-67 & 0.023 & 0.005 & $0.003, r=0.510$ \\
Smoking history & 0.427 & 0.560 & $0.569, r=0.105$ \\
Alcohol history & 0.115 & 0.602 & $0.610, r=0.094$ \\
\hline
\end{tabular}

Notes: ${ }^{2} \mathrm{P}$ value from Fisher's exact test; ${ }^{\mathrm{b}} \mathrm{P}$ value from the Mann-Whitney $U$-test; ${ }^{\mathrm{C}} \mathrm{P}$ value from Spearman rank correlation analysis.

Abbreviation: CTCs, circulating tumor cells.

Table 3 Association Between CTCs And Tumor Markers

\begin{tabular}{|l|l|l|l|}
\hline Parameter & CTC $+(\mathbf{n}=\mathbf{2 3})$ & CTC $-(\mathbf{n}=\mathbf{9})$ & P Value $^{\mathbf{b}}$ \\
\hline CEA $(\mathrm{ng} / \mathrm{mL})$ & $62.47 \pm 22.30^{\mathrm{a}}$ & $10.62 \pm 3.21$ & 0.031 \\
CA199 $(\mathrm{U} / \mathrm{mL})$ & $89.43 \pm 25.09$ & $22.37 \pm 5.77$ & 0.018 \\
\hline
\end{tabular}

Notes: ${ }^{a}$ Mean \pm SEM, ${ }^{b}$ values from the Mann-Whitney test.

Abbreviation: CTCs, circulating tumor cells.

disease progression in a different GC patient. An earlier increment in $\mathrm{E}+\mathrm{CTC}$ count was observed, but the tumor burden was not enlarged on CT image until 90 days later. Furthermore, there was no significant correlation between the dynamic variation trend of E+/Hybrid + CTCs and changes in therapeutic response. This result indicated that monitoring different CTC subtypes exhibits higher sensitivity to evaluate the disease status compared with the traditional methods. Longitudinal tracking of individual patients during the therapeutic process showed a close correlation between the E+CTC count and clinical response. Changes in the proportion of different CTC phenotypes are accompanied by responses to each treatment cycle. Monitoring the CTC phenotypes of patients with metastatic GC may provide further knowledge about disease progression and treatment resistance. However, our present study was limited statistically due to the small sample size and restricted randomness. Thus, the correlation between CTCs and clinical efficacy should be interpreted with caution. Further large-scale clinical trials conducted across multiple clinics over time and an analysis of the overall survival data of patients are needed to evaluate the clinical use (as a predictive marker of survival) of this technique in CTC detection in GC patients. Given the close correlation between CTC subtypes/biomarkers and clinical therapeutic effect, as has been previously reported, ${ }^{30-32}$ we also investigated the relationship between CTC-PD-L1 expression and the clinical response to pembrolizumab in some GC patients. As shown in Figure 4A, in Patient 9 with GC, a sole microsatellite stable (MSS) patient who had a partial response after receiving 2 cycles pembrolizumab regimens, both tumor tissue immunohistochemical (IHC) staining and CTC immunofluorescence staining tested positive for PD-L1. An interesting finding for another MSS responder to pembrolizumab is shown in Figure 4B. The IHC staining showed that the tumor tissue was negative for PD-L1, but the CTC IFC signal was as varied as that of IHC staining. This result may indicate that the expression of CTC PD-L1 may overcome the heterogeneity of tumors and be more representative of the true status of the body. PD-L1-expressing CTCs in GC patients may have some role in the immunophenotypic differential diagnosis of tumors and thereby pinpoint potential candidates for antiPD-1/PD-L1 immune checkpoint therapy. 
a

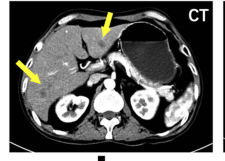

I

SD

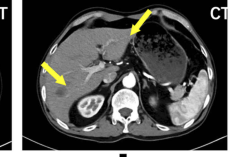

1

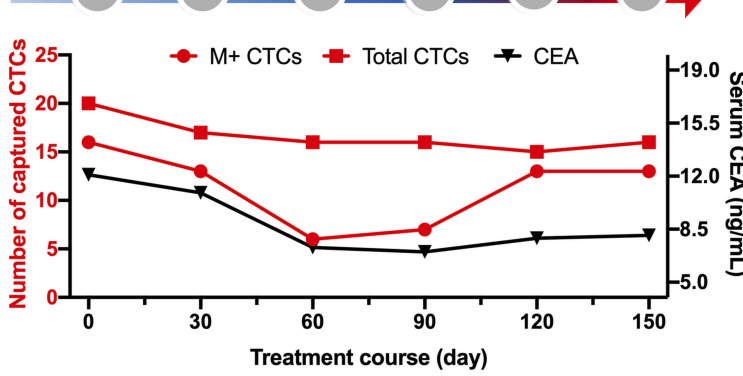

b
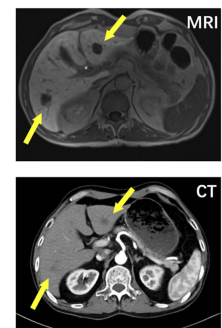

I

PD

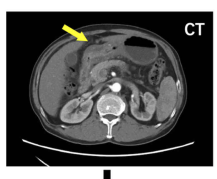

1

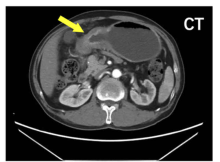

\

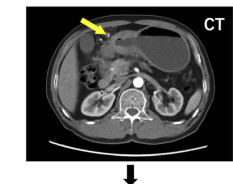

SD

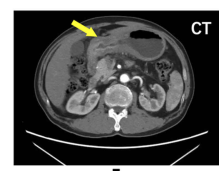

$\downarrow$
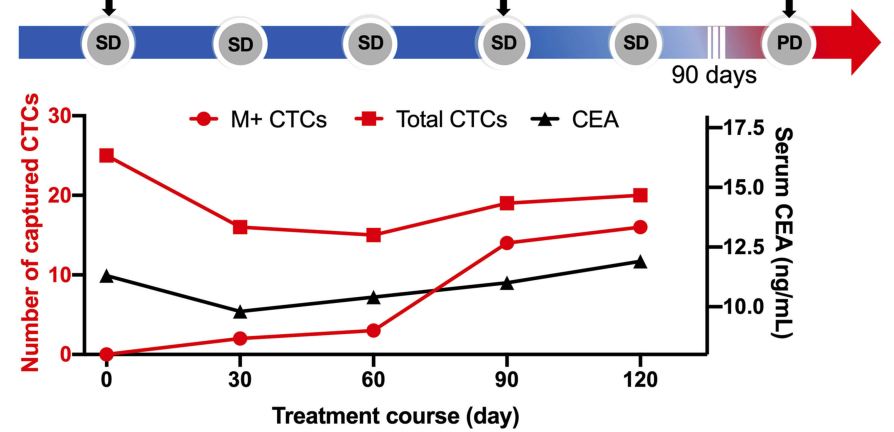

Figure 3 Numerical analysis of 2 patients showing the number of CTCs (total CTCs and E+CTCs, red), concentration of serum CEA (black) (lower panel) and CT/MRI scan (upper panel) in identifying disease progression. Patients with diagnoses and specific therapies shown are (A) gastric adenocarcinoma, SOX: S-I plus oxaliplatin, (B) signetring cell carcinoma, FOLFOX 6: oxaliplatin, 5-FU, and leucovorin. The yellow arrows indicate tumor locations.

Abbreviations: CTCs, circulating tumor cells; E+CTCs, epithelial CTC; CEA, carcinoembryonic antigen; PR, partial response; SD, stable disease; PD, progressive disease.

a

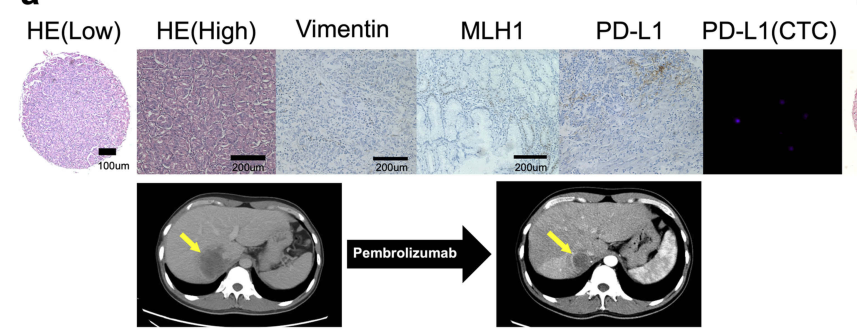

C

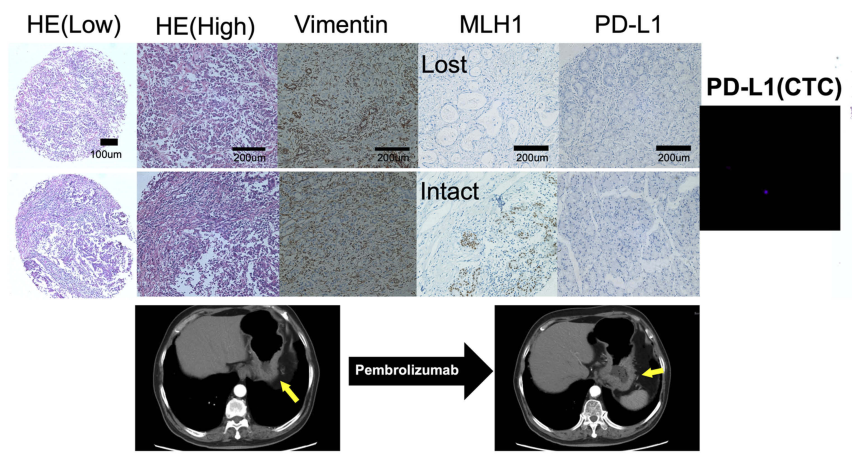

b

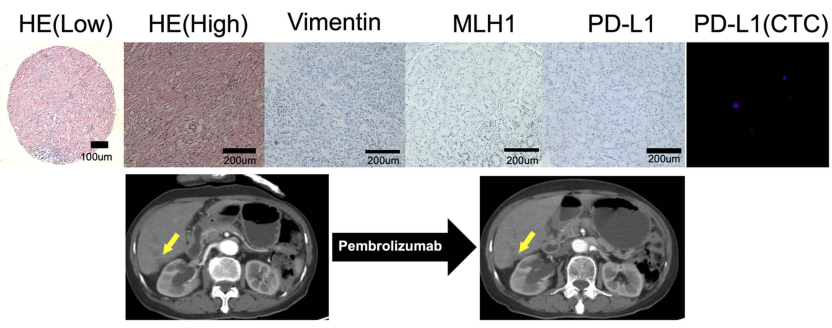

d

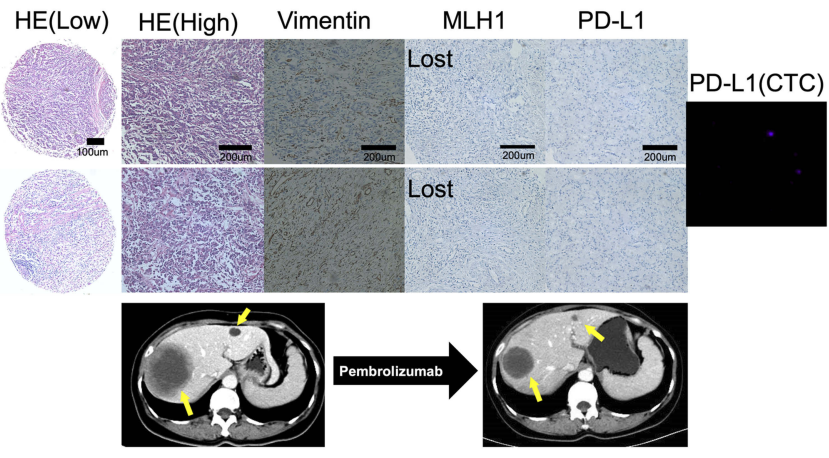

Figure 4 Relationship performance between CTCs-PD-LI/MSI-H and clinical response to pembrolizumab in four GC patients. Patients with IHC, IFC, and CT scan shown are (A) vimentin-, MLHI+, PD-LI+ as well as CTC-PD-LI+, (B) vimentin-, MLH+, PD-LI- as well as CTC-PD-LI+, (C) vimentin+, MLH \pm (heterogeneous MLHI protein expression; lost (upper panel) and intact (lower panel)), PD-LI- as well as CTC-PD-LI-, (D) vimentin-, MLH-, PD-LI- as well as CTC-PD-LI-. CT images before and after pembrolizumab treatment (lower panel). The yellow arrows indicate tumor locations.

Abbreviations: CTCs, circulating tumor cells; PD-LI, programmed cell death-ligand I; MLHI, human mutL homolog I.

As previously reported, ${ }^{30}$ the same phenomenon was also observed in a subtype of GC patients defined as MSI$\mathrm{H}$, who presented a heterogeneous distribution of MSI-H.
As shown in Figure 4C, Patient 16, a sole microsatellite instability high (MSI-H) and PD-L1- patient (both IHC and IFC), exhibited radiographically progressive disease 
according to the RECIST guidelines during pembrolizumab treatment for 3 cycles. Further histologic inspection demonstrated marked geographic heterogeneity of human mutL homolog 1 (MLH1) protein through IHC staining, with both positive and negative tumor regions. This patient had a mixture of MSI-H and MSS regions in the tumor. Instead, as shown in Figure 4D, another patient, with a homogeneous MSI-H status (MLH1+), exhibited a partial response according to the RECIST guidelines. Taken together, these findings contribute to our understanding of how to address the complexity of cancer heterogeneity and reveal how stochasticity in single-cell behaviors promotes phenotypic equilibrium in cancer cell populations. CTCs expressing PD-L1 and MSI are considered costeffective biomarkers that should be routinely detected for immunotherapy in addition to PD-L1 IHC.

\section{Conclusion}

In conclusion, effective application of this technique can allow for CTC quantification and classification for monitoring $\mathrm{GC}$ and predicting the treatment response. CTC enumeration, especially of M+CTCs, was significantly associated with clinical response. A close correlation was observed between the immunophenotypic and therapeutic response of CTCs to immune checkpoint therapy. CTC detection allowed the noninvasive and continuous dynamic monitoring of clinical GC patients, which suggests its potential for individualized treatment and therapeutic evaluation in the future.

\section{Acknowledgments}

The work was supported by National Natural Science Foundation of China (81602489 and 81703767); the Natural Science Foundation of Guangdong (2016A030310242); Sanming Project of Shenzhen (SZSM201612041).

\section{Disclosure}

The authors report no conflicts of interest in this work.

\section{References}

1. Torre LA, Bray F, Siegel RL, Ferlay J, Lortet-Tieulent J, Jemal A. Global cancer statistics, 2012. CA Cancer J Clin. 2015;65(2):87-108. doi: $10.3322 /$ caac. 21262

2. Jamali L, Tofigh R, Tutunchi S, et al. Circulating microRNAs as diagnostic and therapeutic biomarkers in gastric and esophageal cancers. $J$ Cell Physiol. 2018;233(11):8538-8550. doi:10.1002/jep.26850

3. Mirzaei H, Khataminfar S, Mohammadparast S, et al. Circulating microRNAs as potential diagnostic biomarkers and therapeutic targets in gastric cancer: current status and future perspectives. Curr Med Chem. 2016;23(36):4135-4150. doi:10.2174/09298673236661608180 93854
4. Simonian M, Mosallayi M, Mirzaei H. Circulating miR-21 as novel biomarker in gastric cancer: diagnostic and prognostic biomarker. $J$ Cancer Res Ther. 2018;14(2):475. doi:10.4103/0973-1482.175428

5. Chen W, Zheng R, Baade PD, et al. Cancer statistics in China, 2015. CA Cancer J Clin. 2016;66(2):115-132. doi:10.3322/caac.21338

6. Van Cutsem E, Sagaert X, Topal B, Haustermans K, Prenen H. Gastric cancer. Lancet. 2016;388(10060):2654-2664. doi:10.1016/S0140-6736 (16)30354-3

7. Edwards J, Tasker A, Pires Da Silva I, et al. Prevalence and cellular distribution of novel immune checkpoint targets across longitudinal specimens in treatment-naïve melanoma patients: implications for clinical trials. Clin Cancer Res. 2019;25(11):3247-3258. doi:10.1158/10780432.CCR-18-4011

8. Nikolakis G, Brunner M, Boye H, et al. Enlarged mediastinal lymph nodes of a patient with malignant melanoma stage IV under pembrolizumab treatment. Hautarzt. 2019;70(6):443-446. doi:10.1007/ s00105-019-4392-x

9. Tsunoda A, Morikawa K, Inoue T, et al. A prospective observational study to assess PD-L1 expression in small biopsy samples for nonsmall-cell lung cancer. BMC Cancer. 2019;19(1):546. doi:10.1186/ s12885-019-5773-3

10. Oyanagi J, Koh Y, Sato K, et al. Predictive value of serum protein levels in patients with advanced non-small cell lung cancer treated with nivolumab. Lung Cancer. 2019;132:107-113. doi:10.1016/j. lungcan.2019.03.020

11. Katz H, Biglow L, Alsharedi M. Immune checkpoint inhibitors in locally advanced, unresectable, and metastatic upper gastrointestinal malignancies. J Gastrointest Cancer. 2019;118(1):77. doi:10.1007/ s12029-019-00243-8

12. Ding N, Zou Z, Sha H, et al. iRGD synergizes with PD-1 knockout immunotherapy by enhancing lymphocyte infiltration in gastric cancer. Nat Comms. 2019;10(1):1336. doi:10.1038/s41467-019-09296-6

13. Sharma P, Allison JP. The future of immune checkpoint therapy. Science. 2015;348(6230):56-61. doi:10.1126/science.aaa8172

14. Martin-Liberal J, Ochoa de Olza M, Hierro C, Gros A, Rodon J, Tabernero J. The expanding role of immunotherapy. Cancer Treat Rev. 2017;54:74-86. doi:10.1016/j.ctrv.2017.01.008

15. Fuchs CS, Doi T, Jang RW, et al. Safety and efficacy of pembrolizumab monotherapy in patients with previously treated advanced gastric and gastroesophageal junction cancer: phase 2 clinical KEYNOTE-059 trial. JAMA Oncol. 2018;4(5):e180013. doi:10.1001/jamaoncol.2018. 0013

16. Muro K, Chung HC, Shankaran V, et al. Pembrolizumab for patients with PD-L1-positive advanced gastric cancer (KEYNOTE-012): a multicentre, open-label, phase 1b trial. Lancet Oncol. 2016;17 (6):717-726. doi:10.1016/S1470-2045(16)00175-3

17. Kang Y-K, Boku N, Satoh T, et al. Nivolumab in patients with advanced gastric or gastro-oesophageal junction cancer refractory to, or intolerant of, at least two previous chemotherapy regimens (ONO-4538-12, ATTRACTION-2): a randomised, double-blind, placebo-controlled, phase 3 trial. Lancet. 2017;390(10111):2461-2471. doi:10.1016/S0140-6736(17)31827-5

18. Steeg PS. Tumor metastasis: mechanistic insights and clinical challenges. Nat Med. 2006;12(8):895-904. doi:10.1038/nm1469

19. Racila E, Euhus D, Weiss AJ, et al. Detection and characterization of carcinoma cells in the blood. Proc National Acad Sci. 1998;95 (8):4589-4594. doi:10.1073/pnas.95.8.4589

20. Brown HK, Tellez-Gabriel M, Cartron P-F, Vallette FM, Heymann M-F, Heymann D. Characterization of circulating tumor cells as a reflection of the tumor heterogeneity: myth or reality? Drug Discov Today. 2019;24(3):763-772. doi:10.1016/j.drudis.2018.11.017

21. Tsao SC-H, Wang J, Wang Y, Behren A, Cebon J, Trau M. Characterising the phenotypic evolution of circulating tumour cells during treatment. Nat Comms. 2018;9(1):1482. doi:10.1038/s41467018-03725-8 
22. Zieglschmid V, Hollmann C, Böcher O. Detection of disseminated tumor cells in peripheral blood. Crit Rev Clin Lab Sci. 2005;42 (2):155-196. doi:10.1080/10408360590913696

23. Wang S, Zhang C, Wang G, et al. Aptamer-mediated transparent-biocompatible nanostructured surfaces for hepotocellular circulating tumor cells enrichment. Theranostics. 2016;6(11):1877-1886. doi:10.7150/ thno. 15284

24. Zhang N, Deng Y, Tai Q, et al. Electrospun TiO2 nanofiber-based cell capture assay for detecting circulating tumor cells from colorectal and gastric cancer patients. Adv Mater Weinheim. 2012;24(20):27562760. doi:10.1002/adma.201200155

25. Cheng B, He Z, Zhao L, et al. Transparent, biocompatible nanostructured surfaces for cancer cell capture and culture. Int $J$ Nanomedicine. 2014;9(1):2569-2580. doi:10.2147/IJN.S61233

26. Cheng B, Wang S, Chen Y, et al. A combined negative and positive enrichment assay for cancer cells isolation and purification. Technol Cancer Res Treat. 2016;15(1):69-76. doi:10.7785/tcrt.2012.500447

27. Cheng B, Song H, Wang S, et al. Quantification of rare cancer cells in patients with gastrointestinal cancer by nanostructured substrate. Transl Oncol. 2014;7(6):720-725. doi:10.1016/j.tranon.2014.10.001
28. Wu S, Liu S, Liu Z, et al. Classification of circulating tumor cells by epithelial-mesenchymal transition markers. PLoS One. 2015;10(4): e0123976. Samant R, ed. doi:10.1371/journal.pone.0123976

29. Wittekind C. 2010 TNM system: on the 7th edition of TNM classification of malignant tumors. Pathologe. 2010;31(5):331-332. doi:10.1007/s00292-010-1349-3

30. Kim ST, Cristescu R, Bass AJ, et al. Comprehensive molecular characterization of clinical responses to PD-1 inhibition in metastatic gastric cancer. Nat Med. 2018;24(9):1449-1458. doi:10.1038/s41591018-0101-z

31. Goodman CR, Seagle B-L-L, Friedl TWP, et al. Association of circulating tumor cell status with benefit of radiotherapy and survival in earlystage breast cancer. JAMA Oncol. 2018;4(8):e180163. doi:10.1001/ jamaoncol.2018.0163

32. Jordan NV, Bardia A, Wittner BS, et al. HER2 expression identifies dynamic functional states within circulating breast cancer cells. Nature. 2016;537(7618):102-106. doi:10.1038/nature19328

\section{Publish your work in this journal}

OncoTargets and Therapy is an international, peer-reviewed, open access journal focusing on the pathological basis of all cancers, potential targets for therapy and treatment protocols employed to improve the management of cancer patients. The journal also focuses on the impact of management programs and new therapeutic agents and protocols on patient perspectives such as quality of life, adherence and satisfaction. The manuscript management system is completely online and includes a very quick and fair peer-review system, which is all easy to use. Visit http://www.dovepress.com/ testimonials.php to read real quotes from published authors. 